\title{
Li Enrichment, Mass Loss, and CN Abundances in High Rotating K Giants
}

\author{
N.A. Drake, R. de la Reza, L. da Silva \\ Observatório Nacional/MCT, Rua General José Cristino 77, 20921-400, \\ Rio de Janeiro, Brazil \\ D.L. Lambert \\ Department of Astronomy, University of Texas at Austin, Austin, TX \\ 78712 USA
}

\begin{abstract}
High rotating low-mass $\mathrm{K}$ giants can be considered as interesting new "laboratories" for studies of the mixing process and mass loss. By means of high spectral resolution observations of some rapidly rotating $\mathrm{K}$ giants we found a series of connections between rotation, stellar activity, high $\mathrm{Li}$ abundance and mass loss. These giants show low ${ }^{14} \mathrm{~N}$ and high ${ }^{13} \mathrm{C}$ enrichment. Nearly half of them are $\mathrm{Li}$ rich. This frequency is much higher than the $\sim 2 \%$ corresponding to common, low rotating $\mathrm{K}$ giants. They are also the most suitable objects to test new models of rotation-induced mixing or planet engulfing scenarios.
\end{abstract}

\section{Main Results}

High rotating $\left(v \sin i \geq 8.0 \mathrm{~km} \mathrm{~s}^{-1}\right.$ ) low-mass $\mathrm{K}$ giant stars present a number of spectacular properties when compared to the common low rotating $(v \sin i \sim$ $2 \mathrm{~km} \mathrm{~s}^{-1}$ ) $\mathrm{K}$ giants. In the sample of high rotating $\mathrm{K}$ giants studied by Drake et al. (2002), some stars have a $\mathrm{Li}$ abundance larger than the ISM value as in the case of $\operatorname{HDE} 233517\left(v \sin i=17.6 \mathrm{~km} \mathrm{~s}^{-1}\right)$ and HD $9746\left(v \sin i=8.7 \mathrm{~km} \mathrm{~s}^{-1}\right)$ (Balachandran et al. 2000). An interesting high velocity and moderate rotation $\mathrm{K}$ giant $\mathrm{PDS} 68\left(v \sin i=6.0 \mathrm{~km} \mathrm{~s}^{-1}\right)$ presenting a very large Li abundance equal to $\log \varepsilon(\mathrm{Li})=3.9$ has been included in our sample too.

Our detailed study of another Li-rich K giant, PDS $365\left(v \sin i=20 \mathrm{~km} \mathrm{~s}^{-1}\right)$, showed that this star appears to present $\mathrm{C} / \mathrm{N}$ ratios larger than those expected for a "normal" giant that has already passed the first dredge-up phase. In this respect, it is interesting to remark that similar $\mathrm{C}$ and $\mathrm{N}$ anomalies appear also to be present in PDS $100\left(v \sin i=9 \mathrm{~km} \mathrm{~s}^{-1}\right.$ ) (Reddy et al. 2002) and HD 9746 (Berdyugina \& Savanov 1994) which are the only Li-rich rapidly-rotating giants with $\mathrm{CNO}$ values known in the literature. The difference between observed and predicted values of ${ }^{12} \mathrm{C} /{ }^{14} \mathrm{~N}$ ratios depends essentially on the mass of the star. In any case, if a rotation-induced mixing is in action, this is not influencing the ${ }^{14} \mathrm{~N}$ surface abundance, but enhancing the ${ }^{13} \mathrm{C}$ abundance. The ${ }^{12} \mathrm{C} /{ }^{13} \mathrm{C}$ ratios of these high rotating Li-rich giants are in general lower than those of the standard theory independently of a stellar mass in the $(1-2) \mathrm{M}_{\odot}$ range.

High rotating Li-rich giants have high mass loss rates as shown by their IR excesses measured by IRAS. The distribution of the IRAS colors of the high 
rotating giants allowed us to infer that the association of rapid rotation with lithium enrichment appears when a high far-IR excess is present. The signature of mass loss can also be found in some spectral features ( $\mathrm{Na} \mathrm{D}$ and $\mathrm{H} \alpha$ lines).

Two main classes of astrophysical scenarios attempt to explain the existence of high rotating Li-rich giants.

I. Pure internal process. Recently, Palacios et al. (2001) proposed a scenario based on rotation-induced mixing due to an important energy release in a lithium-burning shell. According to this model, surface Li enrichment in lowmass $\mathrm{K}$ giant occurs at the bump in the luminosity function on the RGB. This mechanism predicts also an enhancement of mass loss and dust shell formation as found by de la Reza et al. (1996, 1997).

II. Planet engulfing process. Siess \& Livio (1999) developed the idea that a RGB star can engulf a planet or a brown dwarf, and thus increase its surface Li abundance. The planet engulfing produces also an enhancement of the stellar angular momentum and, hence, an increasing of the stellar rotation velocity, as well as a mass loss. The main difficulty of this scenario lies in the fact that the $\mathrm{Li}$ enrichment of the giant's surface is limited by the planet $\mathrm{Li}$ abundance, which is similar to that of ISM. Trying to overcome this problem, Denissenkov \& Weiss (2000) proposed that planet ingestion can trigger the ${ }^{7} \mathrm{Be}$ mechanism, producing then more ${ }^{7} \mathrm{Li}$. At the same time, the enhanced star rotation increases rotationinduced mixing. Nevertheless, the corresponding mass loss is much delayed. The drawback of both versions of this scenario is the fact that Li enrichment can take place anywhere during the RGB phase and this is not observed. Li enrichment appears only at the RGB bump or later (Charbonnel \& Balachandran 2000).

A detection of ${ }^{6} \mathrm{Li}$ would give support to a recent engulfing event due to the fragility of this isotope. However, no ${ }^{6} \mathrm{Li}$ has been found until now in Li-rich giants (Balachandran et al. 2000; Reddy et al. 2002; Drake et. al. 2002).

\section{References}

Balachandran, S.C., Fekel, F.C., Henry, G.W., \& Uitenbroek, H. 2000, ApJ 542, 978

Berdyugina, S.V., \& Savanov, I.S. 1994, Astron. Lett. 20, 639

Charbonnel, C., \& Balachandran, S.C. 2000, A\&A 359, 563

de la Reza, R., Drake, N.A., \& da Silva, L. 1996, ApJ 456, L115

de la Reza, R., Drake, N.A., da Silva, L., Torres, C.A.O., \& Martin, E.L. 1997, ApJ 482, L77

Denissenkov, P.A., \& Weiss, A. 2000, A\&A 358, L49

Drake, N.A., de la Reza, R., da Silva, L., \& Lambert, D.L. 2002, AJ 123, 2703

Palacios, A., Charbonnel, C., \& Forestini, M. 2001, A\&A 375, L9

Reddy, B. E., Lambert, D.L., Hrivnak, B. J., \& Bakker, E. J. 2002, AJ 123, 1993

Siess, L., \& Livio, M. 1999, MNRAS 308, 1133 